\title{
Redefining prognostication of de novo cytogenetically normal acute myeloid leukemia in young adults
}

\author{
Sze P. Tsui ${ }^{1}$, Ho W. Ip ${ }^{1}$, Nicole Y. Saw ${ }^{2}$, Chunxiao Zhang ${ }^{3}$, Arthur K. Cheung ${ }^{4,5}$, Nelson K. Ng ${ }^{3}$, Cheuk H. Man ${ }^{3}$, \\ Stephen S. Lam $\mathbb{B}^{3}$, Wing F. Tang ${ }^{1}$, Chi H. Lin ${ }^{4}$, Grace H. Cheng ${ }^{4}$, Chun H. Au', Edmond S. Ma ${ }^{6}$, Tsun L. Chan ${ }^{6}$, \\ Jason C. So ${ }^{7}$, Margaret H. Ng ${ }^{8}$, Kelvin C. Cheng ${ }^{8}$, Kit F. Wong9 ${ }^{9}$, Lai P. Siu', Sze F. Yip ${ }^{10}$, Shek Y. Lin ${ }^{11}$, June S. Lau ${ }^{12}$, \\ Tsan H. Luk ${ }^{12}$, Harold K. Lee ${ }^{13}$, Chi K. Lau ${ }^{14}$, Bonnie Kho ${ }^{15}$, Joycelyn P. Sim³, Yok L. Kwong $\mathbb{1}^{3}$, Suet Y. Leung ${ }^{4,5,16}$, \\ Asif Javed (1D) $2,4,5$ and Anskar Y. Leung $\mathbb{B}^{3,5}$
}

\section{Dear Editor,}

About $50 \%$ of acute myeloid leukemia (AML) showed normal cytogenetics $(\mathrm{CN})$ with leukemogenesis driven putatively by recurrent mutations. These mutations occur singly or in combination, as dominant clones or subclones $^{1-4}$. Induction with daunorubicin and cytarabine is the standard for young and fit patients, achieving first complete remission (CR1) in 60-80\% cases. Postremission strategies include consolidation with highdose cytarabine and allogeneic hematopoietic stem cell transplantation (allo-HSCT). The latter may reduce the risk of relapse but is associated with mortality and longterm morbidities. The European LeukemiaNet (ELN) guidelines, based on cytogenetic and genetic risk stratification, provide general recommendations on prognostication and allo-HSCT for $\mathrm{AML}^{5}$. We performed next-generation sequencing (NGS) for young patients with de novo CN-AML, diagnosed between 2003 and 2019 , who were treated with a relatively uniform algorithm to examine the prognostic impact of mutation combinations. Machine learning was used to generate prediction model and its performance was compared with that based on ELN guidelines. Clinical treatment and methodology are described in Supplemental Materials (see also Supplemental Fig. S1).

\footnotetext{
Correspondence: Asif Javed (javed@hku.hk) or

Anskar Y. Leung (ayhleung@hku.hk)

${ }^{1}$ Department of Pathology, Queen Mary Hospital, Hong Kong SAR, China

${ }^{2}$ School of Biomedical Sciences, LKS Faculty of Medicine, The University of Hong Kong, Hong Kong SAR, China

Full list of author information is available at the end of the article
}

Four hundred and fifty-nine patients with de novo $\mathrm{CN}$ AML, at a median age of 49 years (range: 18-60 years), were studied (Supplemental Table S1). Their treatment outcomes are shown in Fig. 1A. Four hundred and thirtysix patients received induction chemotherapy, of whom 419 patients $(96 \%)$ received standard " $7+3$ " regimen and 17 (4\%) received " $5+2$ ", idarubicin, mitoxantrone, hypomethylating agents, or homoharringtonine-based regimens. After the first induction, $\mathrm{CR} / \mathrm{CRi}$ (CR with incomplete hematological recovery) was achieved in 283 patients $(65 \%)$. There was no significant difference in leukemia-free survival (LFS) (95\% confidence interval (C. I.) $0.92-1.55 ; P=0.18$ ) or overall survival (OS) (95\% C.I. $0.99-1.80 ; P=0.06$ ) between patients who achieved CR or $\mathrm{CRi}$ after first induction and they were analyzed together. High-dose daunorubicin $\left(90 \mathrm{mg} / \mathrm{m}^{2}\right)$ was associated with significantly higher chance of CR compared with standard dose $\left(60 \mathrm{mg} / \mathrm{m}^{2}\right.$; Supplemental Table S2). Patients who failed first induction received salvage chemotherapy (Supplemental Materials), resulting in CR1 in another 113 patients. Post-remission therapy included high-dose cytarabine, with some patients having received 1-2 courses of " $5+2$ " before it. Allo-HSCT was performed in 181 patients from different donor types (HLA identical siblings, $N=103$; matched unrelated, $N=75$; haploidentical, $N=1$; identical twins, $N=2$ ).

For the initial 187 patients analyzed by the pan-cancer panel (Supplemental Data 1), mutations were identified in 77 genes, with 42 genes mutated in $\geq 1 \%$ and 13 genes mutated in $\geq 5 \%$ of patients (median: 3 mutations per patient; range: 0-7). Subsequently, 43 patients were 


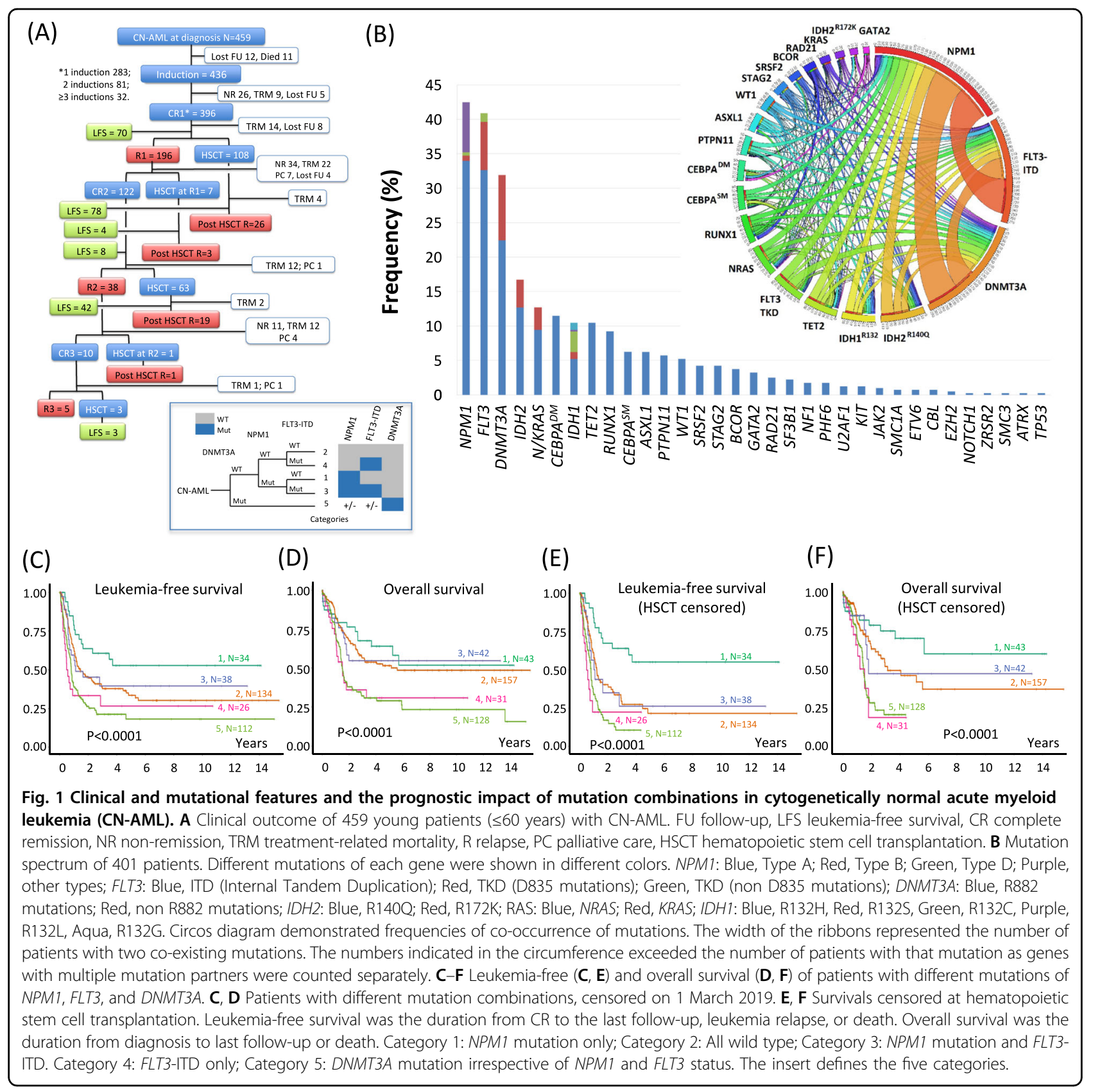

analyzed by the myeloid-focused panel, 33 patients by the Trusight panel, and 138 patients by the AML panel. In these 214 patients, mutations were identified in 29 genes (median: 3 mutations per patient; range: 0-6). Mutations were categorized according to their putative functions in hematopoiesis or leukemogenesis (Supplemental Table S3). The most frequently mutated genes are shown in Fig. 1B. NPM1, DNMT3A, CEBPA ${ }^{\mathrm{DM}}$, and $I D H 1 / 2$ often showed variant allele frequency (VAF) of 40-50\%; whereas FLT3-ITD, NRAS, and FLT3-TKD showed more heterogeneous VAF of $10-50 \%$ (Supplemental Fig. S2).
NPM1 and $C E B P A^{\mathrm{DM}}$ mutations were associated with superior CR/CRi rates and RUNX1 and ASXL1 mutations with inferior CR/CRi rates after first induction (Supplemental Table S4). To examine the factors affecting survivals, age, gender, white blood cell count (WCC), daunorubicin dose (60 versus $90 \mathrm{mg} / \mathrm{m}^{2}$ ), achievement of $\mathrm{CR} / \mathrm{CRi}$ after induction or salvage chemotherapy, alloHSCT at CR1 as well as individual gene mutations were analyzed by univariate analysis. Age and WCC varied with LFS, event-free survival (EFS), and OS as continuous functions and were defined as numerical data (Supplemental Fig. S3). High-dose daunorubicin and HSCT at 
CR1 were associated with superior LFS, EFS, and OS and achievement of CR/CRi was associated with superior EFS and OS, whereas high WCC and FLT3-ITD and DNMT3A mutations were associated with inferior LFS, EFS, and OS (Supplemental Table S5A). High-dose daunorubicin appeared to negate the adverse prognosis of DNMT3A mutations, consistent with previous reports ${ }^{6}$ (Supplemental Fig. S4). Subsequently, these factors were evaluated in multivariate analysis. The prognostic impacts of FLT3-ITD and DNMT3A mutations, achievement of $\mathrm{CR} / \mathrm{CRi}$, and HSCT at CR1 have remained unchanged but those of high-dose daunorubicin have become largely insignificant (Supplemental Table S5B). NPM1 mutation was associated with superior LFS and EFS but not OS.

NPM1, DNMT3A, and FLT3-ITD were further evaluated for their relative impacts on LFS (Supplemental Fig. S5) and OS (Supplemental Fig. S6). DNMT3A mutation negated the prognostic impact of NPM1 mutation and FLT3-ITD, attesting to its overriding impact on prognosis amidst co-existing mutations. FLT3-ITD also negated the prognostic impact of NPM1 but not DNMT3A mutation. NPM1 mutation had no significant impact on the adverse prognostic effects of DNMT3A mutation and FLT3-ITD. Their combinations showed variable LFS and OS (Supplemental Fig. S7) and were further categorized into five groups (Supplemental Table S6). Sole NPM1 mutation (Category 1) showed superior LFS and OS while sole FLT3-ITD (Category 4) and presence of DNMT3A mutation (Category 5) showed inferior LFS and OS. Patients of wild type for all 3 genes (Category 2) and with co-existing NPM1 mutation and FLT3-ITD (Category 3) showed intermediate LFS. However, their OS were indistinguishable from that of Category 1 (Fig. 1C, D). When outcomes were censored at HSCT, Category 1 remained superior, Categories 2 and 3 were intermediate, and Categories 4 and 5 remained inferior (Fig. 1E, F). Subgroup analyses were performed to evaluate the prognostic impact of other recurrent mutations on the five categories. IDH1R132H was associated with inferior LFS and OS in Category 2 exclusively (Supplemental Fig. S8). Other mutations had no significant impact on these categories or their occurrences were too low for comparison (Supplemental Table S7).

To examine whether prognostication by ELN 2017 guidelines might apply to young patients with CN-AML, the present cohort was classified according to the stipulated risk groups, based exclusively on gene mutations. High FLT3-ITD was defined by VAF $\geq 0.33$, corresponding to an allelic ratio of $\geq 0.5$ (Supplemental Table S8). There was a trend toward a difference in LFS and OS among the three risk groups. However, it was statistically insignificant (Fig. 2A, B). We examined the impact of $D N M T 3 A$ mutation on each ELN-defined risk groups in our patients. DNMT3A mutation negatively impacted on
LFS and OS in the favorable (Supplemental Fig. S9A, B) and intermediate risk groups (Supplemental Fig. S9C, D) but not in the unfavorable risk group (Supplemental Fig. S9E, F). Incorporating DNMT3A mutation into the ELN risk categorization as an unfavorable risk factor separated the three risk groups and significantly improved the risk stratification (Fig. 2C, D).

The genetic makeup of leukemic clones was extremely diverse (Fig. 2E and Supplemental Fig. S10). Of the 401 patients on whom NGS was performed, 383 patients showed detectable mutation of genes in the AML panel with at least 217 distinct clonal subtypes. The most common subtypes comprised co-dominant NPM1 and $D N M T 3 A$ mutations, usually in conjunction with other co-dominant or subclone mutations. Sole NPM1 $(1.00 \%)$ or DNMT3A mutations $(0.50 \%)$ or their codominance without subclones $(0.50 \%)$ were relatively uncommon. NPM1 mutation was infrequently found in subclones, and in those rare circumstances, the dominant clones were mostly DNMT3A or IDH2R $140 \mathrm{Q}$ mutations. FLT3-ITD occurred most frequently as subclones. However, in $2.74 \%$ patients, FLT3-ITD occurred as the sole mutation, suggesting its role as leukemic driver early in the leukemic hierarchy ${ }^{7}$. CEB$P A^{\mathrm{DM}}$ occurred predominantly as a sole mutation in $5.24 \%$ patients. Forty-six patients $(11.47 \%)$ were negative for all common or ELN risk-defining mutations, viz. NPM1, DNMT3A, FLT3, IDH1/2, CEBPA, ASXL1, RUNX1, and TP53. They showed rare mutations, some of which, including those of spliceosome genes ${ }^{8}$, were dominant and sole mutations, suggesting pathogenetic role in leukemogenesis (Supplemental Materials, Supplemental Fig. S11, and Supplemental Table S9).

The database built up in this study formed a foundation for the development of prediction model (https:// redefiningprognosis.shinyapps.io/denovo_cnaml/) that might inform clinical decision. Its application was highlighted by two hypothetical patients (Fig. 2F). The information provided quantitative measurement of survival benefits of individual patients based on their demographics and genotypes. Its performance was compared with that of the ELN risk stratification-based model based on concordance index. Using the present cohort of 401 patients as a training set, our prediction model showed a $4.6 \%$ higher concordance over the ELNbased model (Fig. 2G). To validate these models, a subset of The Cancer Genome Atlas patients comprising $83 \mathrm{de}$ novo $\mathrm{CN}$-AML patients aged $\leq 60$ years was used as a validation cohort. Patients who received HSCT at refractory stage were not included as they were not represented in the training set. Again, our model showed 6.45\% higher concordance over ELN-based model. The difference in concordance in both cohorts was statistically significant. We proposed that this multistage model 


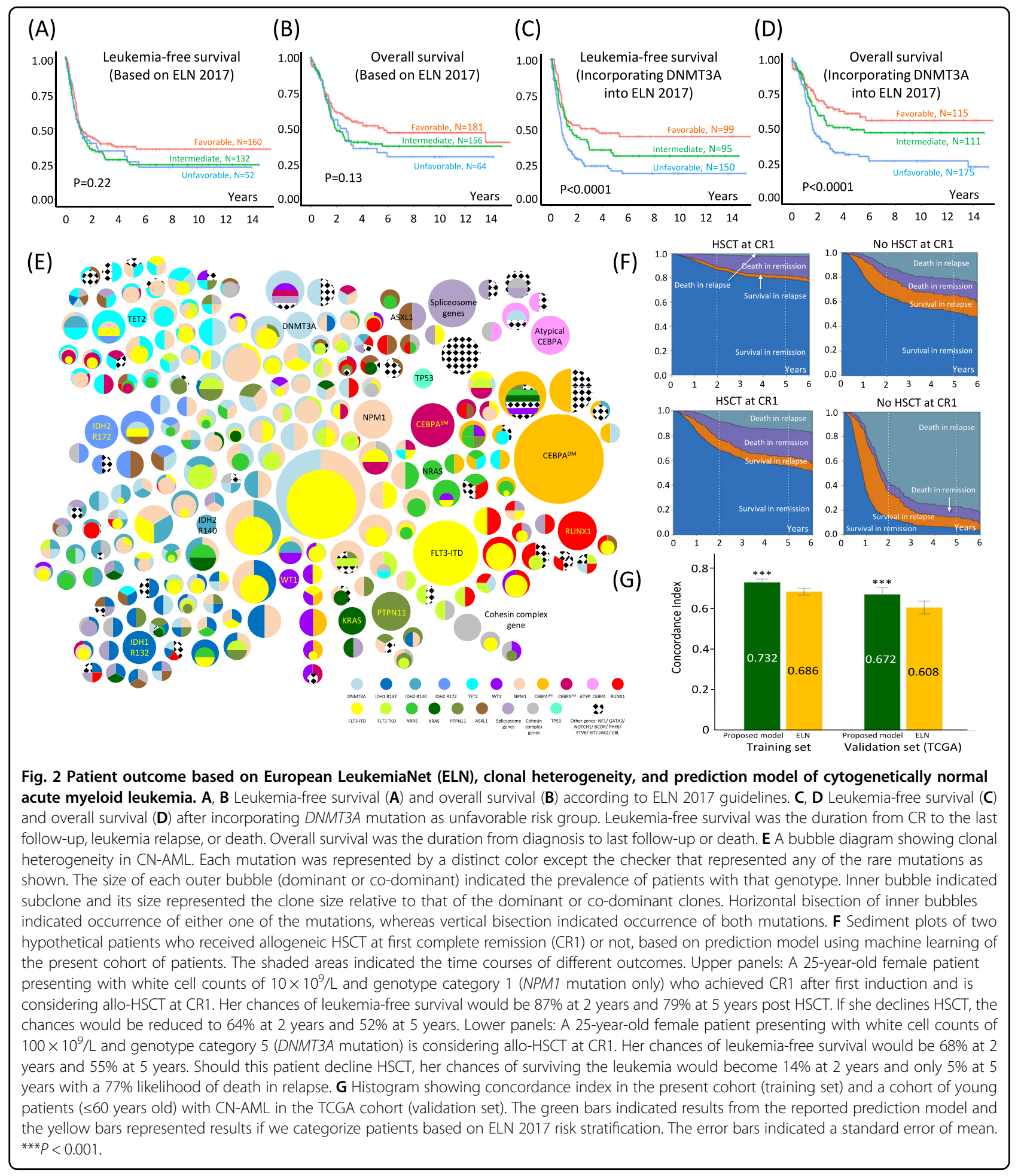

might provide more personalized guidance to inform post-remission therapy with particular reference to allo$\mathrm{HSCT}^{9}$. Our findings corroborated with recent reports demonstrating room to refine risk stratification based on sequencing and transcriptomic results of patients enrolled into clinical trials ${ }^{10}$.
In conclusion, in young patients with de novo CN-AML who received conventional induction chemotherapy, consolidation, and allo-HSCT, incorporation of DNMT3A mutation into risk stratification significantly improved their prognostication. Predication model based on machine learning of our database generated a more 
personalized tool to guide post-remission therapy. The diverse clonal heterogeneity and the pathogenetic significance of mutation combinations provided important leads for future mechanistic study.

\section{Acknowledgements}

The works were supported by the Croucher Senior Medical Research Fellowship (260960158), S.K. Yee Medical Foundation (260940149, 260940187), Li Shu Fan Medical Foundation, Collaborative Research Fund (C7028-19G) and Theme-Based Research Scheme (T12-702/20-N), The Hong Kong Jockey Club Charities Trust and LKS Faculty of Medicine.

\section{Author details}

'Department of Pathology, Queen Mary Hospital, Hong Kong SAR, China. ${ }^{2}$ School of Biomedical Sciences, LKS Faculty of Medicine, The University of Hong Kong, Hong Kong SAR, China. ${ }^{3}$ Division of Haematology, Department of Medicine, LKS Faculty of Medicine, The University of Hong Kong, Pokfulam, Hong Kong SAR, China. ${ }^{4}$ Centre for PanorOmic Sciences, LKS Faculty of Medicine, The University of Hong Kong, Pokfulam, Hong Kong SAR, China. ${ }^{5}$ The Jockey Club Centre for Clinical Innovation and Discovery, LKS Faculty of Medicine, The University of Hong Kong, Pokfulam, Hong Kong SAR, China. ${ }^{6}$ Department of Pathology, Hong Kong Sanatorium and Hospital, Hong Kong SAR, China. 'Department of Pathology, Hong Kong Children's Hospital, Hong Kong SAR, China. ${ }^{8}$ Department of Anatomical and Cellular Pathology, The Chinese University of Hong Kong, Hong Kong SAR, China. ${ }^{9}$ Department of Pathology, Queen Elizabeth Hospital, Hong Kong SAR, China. ${ }^{10}$ Department of Pathology, Tuen Mun Hospital, Hong Kong SAR, China. ${ }^{11}$ Department of Medicine and Geriatrics, United Christian Hospital, Hong Kong SAR, China. ${ }^{12}$ Department of Medicine, Queen Elizabeth Hospital, Hong Kong SAR, China. ${ }^{13}$ Department of Medicine, Princess Margaret Hospital, Hong Kong SAR, China. ${ }^{14}$ Department of Medicine, Tseung Kwan O Hospital, Hong Kong SAR, China. ${ }^{15}$ Department of Medicine, Pamela Youde Nethersole Eastern Hospital, Hong Kong SAR, China. ${ }^{16}$ Department of Pathology, LKS Faculty of Medicine, The University of Hong Kong, Pokfulam, Hong Kong SAR, China

\section{Data availability}

The datasets generated and analyzed during the current study are available from the corresponding author on reasonable request.

\section{Conflict of interest}

The authors declare that they have no conflict of interest.

\section{Publisher's note}

Springer Nature remains neutral with regard to jurisdictional claims in published maps and institutional affiliations.

Supplementary Information accompanies this paper at (https://doi.org/ 10.1038/s41408-020-00373-4).

Received: 1 August 2020 Revised: 21 September 2020 Accepted: 2 October 2020

Published online: 19 October 2020

\section{References}

1. Ley, T. J. et al. Genomic and epigenomic landscapes of adult de novo acute myeloid leukemia. N. Engl. J. Med. 368, 2059-2074 (2013).

2. Papaemmanuil, E. et al. Genomic classification and prognosis in acute myeloid leukemia. N. Engl. J. Med. 374, 2209-2221 (2016).

3. Rose, D. et al. Subtype-specific patterns of molecular mutations in acute myeloid leukemia. Leukemia 31, 11-17 (2017).

4. Metzeler, K. H. et al. Spectrum and prognostic relevance of driver gene mutations in acute myeloid leukemia. Blood 128, 686-698 (2016).

5. Döhner, H. et al. Diagnosis and management of AML in adults: 2017 ELN recommendations from an international expert panel. Blood 129, 424-448 (2017).

6. Patel, J. P. et al. Prognostic relevance of integrated genetic profiling in acute myeloid leukemia. N. Engl. J. Med. 366, 1079-1089 (2012).

7. Garg, M. et al. Profiling of somatic mutations in acute myeloid leukemia with FLT3-ITD at diagnosis and relapse. Blood 126, 2491-2501 (2015).

8. Gerstung, M. et al. Precision oncology for acute myeloid leukemia using a knowledge bank approach. Nat. Genet. 49, 332-340 (2017).

9. Bamopoulos, S. A. et al. Clinical presentation and differential splicing of SRSF2, U2AF1 and SF3B1 mutations in patients with acute myeloid leukemia. Leukemia 34, 2621-2634 (2020).

10. Herold, T. et al. Validation and refinement of the revised 2017 European LeukemiaNet genetic risk stratification of acute myeloid leukemia. Leukemia https:/doi.org/10.1038/s41375-020-0806-0 (2020). 